\title{
Influence du changement climatique sur la diversité inter et intra-spécifique des plantes cultivées à Tougou au nord du Burkina Faso
}

\author{
Dasmané BAMBARA*, Ablassé BILGO, François LOMPO et Victor HIEN \\ Institut de l'Environnement et de Recherches Agricoles (INERA), 01 BP 476 Ouagadougou 01, \\ Burkina Faso. \\ *Auteur correspondant, E-mail : das.bambara@coraf.org ; Tél : 22670285245
}

\section{RESUME}

L'objectif de l'étude est d'évaluer la diversité taxonomique et la variabilité inter et intra-spécifique des plantes cultivées non spontanées dans un contexte de changement climatique. Les données sont collectées à l'aide d'une enquête au près de 75 producteurs menée suivant des interviews semi-structurées. Les résultats ont révélé l'existence de 122 écotypes appartenant à 49 espèces, 39 genres et 22 familles. La diversité interspécifique représentait $73 \%$ de la diversité des plantes cultivées au plan national. La variabilité intraspécifique était faible comparée à la variabilité au plan national. Elle était plus élevée chez les céréales et les cultures potagères pluviales avec 2 à 10 écotypes par espèce contre 1 à 3 écotypes par espèce chez les cultures maraîchères. Les écotypes ayant un cycle de plus de 90 jours représentant 10,5\% de l'ensemble des écotypes recensés, sont menacés de disparition à cause du changement climatique. Une stratégie de collecte, de conservation, d'amélioration et de promotion de ces ressources ainsi que la restauration des écosystèmes dégradés s'avèrent nécessaire pour le maintien de la phytodiversité cultivée.

(C) 2011 International Formulae Group. All rights reserved.

Mots clés : Burkina Faso, agro biodiversité, écotype, espèces menacées, conservation.

\section{INTRODUCTION}

Le changement climatique qui se manifeste par l'avènement plus fréquent de phénomènes climatiques extrêmes (inondation, sécheresse, vents violents, etc.) entraîne le déplacement des isohyètes du Nord vers le Sud avec comme conséquence une modification du cadre écologique (IPCC, 2007). L'agriculture est affectée négativement par ce changement climatique (Niang, 2009), en particulier dans les pays en développement (Nelson et al., 2009). Une des stratégies adoptées par les agriculteurs pour faire face aux conséquences de ce changement climatique est la diversification des productions végétales (Lo et Kaere, 2009) qui passe par la préservation des espèces végétales (FAO, 2008; Naeem et Defries, 2009). La diversité biologique maintient la vie sur terre grâce à ses rôles écologiques et de pourvoyeur de produits de subsistance pour les êtres vivants (FAO, 2008). Au Burkina Faso, la diversité biologique, dans son état actuel, connaît un appauvrissement du fait des effets conjugués de la détérioration des conditions climatiques et de la dégradation 
des écosystèmes (Naeem et Defries, 2009). La préservation des espèces est pourtant un impératif pour tout développement durable (Soulama, 2010). Cela passe nécessairement par une bonne connaissance de cette diversité biologique (Belem-Ouédraogo, 1998). A l'instar de tous les pays de la sous-région de l'Afrique de l'Ouest, le Burkina Faso a fait de cette connaissance un moyen de son développement (CAPES, 2006). De nombreux auteurs ont contribué de manière appréciable à la connaissance de la flore et de la végétation d'ensemble du pays (Guinko, 1984b ; Lebrun et al., 1991; Ouédraogo, 2006). D'autres travaux, portant sur des catégories ou des groupes taxonomiques de plantes (Thiombiano, 2005) et sur des territoires à petite échelle (Hahn-Hadjali, 1997 ; Neumann et Müller-Haude, 1999 ; Devineau, 2001) ont considérablement amélioré les connaissances sur les ressources végétales. Malgré toutes ces contributions, la diversité biologique du Burkina Faso n'est pas encore suffisamment connue car des inventaires systématiques restent à réaliser (CAPES, 2006) pour certaines catégories de plantes. C'est particulièrement le cas de la diversité biologique des plantes cultivées qui est affectée par un environnement climatique changeant et dont on se demande si les pratiques séculaires peuvent toujours permettre de la conserver (Baco et al., 2008). Ce contexte menace l'évolution vers une société durable (CTA, 2009). Depuis la convention sur la diversité biologique (CDB), l'évaluation de la richesse variétale est devenue un préalable à toute tentative de conservation in-situ (Baco et al., 2008). Paradoxalement, il n'existe pas suffisamment d'informations sur la connaissance actuelle de la diversité des espèces végétales cultivées. Certes, il y a eu des inventaires et des collectes d'espèces cultivées aux niveaux régional et national (Guinko, 1984a; Sié et al., 1998 ; Balma et al., 2004 ; BarroKondombo et al., 2008) qui ont apporté un éclaircissement sur les écotypes cultivés au Burkina Faso, mais la littérature sur la diversité inter et intra-spécifique des plantes cultivées au niveau local (village) reste insuffisante (CAPES, 2006). L'échelle locale paraît pourtant être l'espace géographique et socio-économique pertinent pour une évaluation de la diversité des espèces cultivées compte tenu du caractère endémique de certaines espèces végétales (Naeem et Defries, 2009) et de la grande variabilité à petite échelle du climat de la zone tropicale (Paturel et al., 1996). L'objectif de l'étude est d'évaluer à travers des enquêtes et des observations, la diversité taxonomique et la variabilité inter et intra-spécifique des plantes cultivées, puis d'identifier les espèces menacées de disparition pour des raisons climatiques dans le village de Tougou au nord du Burkina Faso.

\section{MATERIEL ET METHODES Milieu d'étude}

L'étude est menée dans le village de Tougou, situé entre les parallèles $13^{\circ} 11^{\prime}$ et $13^{\circ} 26^{\prime}$ de latitude Nord et les méridiens $2^{\circ} 6^{\prime}$ et $2^{\circ} 24^{\prime}$ de longitude Ouest (Figure 1). De groupe socioculturel essentiellement mossi, la population de Tougou comptait 4574 habitants en 2004 dont 2144 hommes et 2430 femmes (INSD, 2008). La densité de la population est de 114 habitants $/ \mathrm{km}^{2}$. Les activités socio-économiques comprennent l'agriculture, l'élevage, la pêche, le commerce, l'apiculture, etc. Le climat est de type subsahélien avec une pluviosité moyenne annuelle de 500 à $700 \mathrm{~mm}$ (Koussoubé et al., 2006) étalée sur 4 mois, de juin à septembre. La répartition des pluies est irrégulière dans le temps et dans l'espace. La température maximale varie entre 30 et $38{ }^{\circ} \mathrm{C}$ durant la saison des pluies et peut atteindre $43{ }^{\circ} \mathrm{C}$ en avril et mai, en saison sèche. L'évapotranspiration potentielle est de 2247 mm en années sèches et de $1693 \mathrm{~mm}$ en années humides. Au cours des trois dernières décennies (1980-2009), le changement climatique s'est manifesté dans la zone d'étude par une instabilité des paramètres climatiques essentiels. Tandis qu'on assiste à 
une baisse globale de la pluviométrie, la température, la vitesse du vent et l'évapotranspiration sont globalement en hausse (Bambara, 2011). L'humidité relative et la durée d'insolation sont restées instables de 1980 à 2009. La végétation naturelle comprend deux types de formations: la savane et la steppe. La flore de la savane est dominée par Balanites aegyptiaca (L.) Del., Cassia sieberana DC., Sclerocarya birrea (A. Rich.) Hochst., Vitellaria paradoxa C.F.Gaertn tandis que dans la steppe les principales espèces comprennent Combretum micranthum G. Don, Combretum glutinosum Perr., Guiera senegalensis J.F. Gmel. Le tapis herbacé est dominé par Cymbopogon schoenanthus (L.) Spreng., Cassia tora (L.) Haines et Schoenefeldia gracilis Kunth (Bambara, 2011). Le contexte écologique, caractérisé par la dégradation des sols, du couvert végétal et l'insuffisance pluviométrique, a amené les producteurs à adopter diverses techniques de production pour minimiser les risques de mauvais rendements à la récolte. La diversification des espèces et des variétés végétales fait partie de ces stratégies de production adaptatives (Koussoubé et al., 2006).

\section{Méthodologie}

Les espèces et les écotypes sont recensés à partir des entretiens de groupes et de collecte d'échantillons de semences. La méthode d'enquête a consisté en des interviews directes semi-structurées et conduites selon les deux étapes suivantes : la pré-enquête et l'enquête formelle.

\section{Pré-enquête}

La pré-enquête, visant à recenser toutes les espèces végétales cultivées dans le village, est conduite comme une enquête informelle mais en une assemblée générale de 75 producteurs. Le recensement des espèces a été fait de façon participative par filière végétale avec l'ensemble des producteurs. Six filières ont été constituées en fonction des groupements de producteurs présents dans le village (Tableau 1). Toutes les personnes interrogées étaient des membres de ces groupements. Les espèces végétales ont été décrites à travers leur nom commun, leur nom scientifique, leur famille botanique d'appartenance, l'origine de la plante, le nombre d'écotypes de l'espèce.

\section{Enquête formelle et collecte de semences écotypiques}

L'enquête formelle ayant pour objectif d'inventorier et de décrire tous les écotypes des différentes espèces végétales recensées lors de la pré-enquête, a consisté en des interviews semi-structurées organisées en groupes mixtes de producteurs et productrices afin d'éviter les biais liés à la dénomination paysanne des écotypes. Six groupes ont été constitués selon les filières de production végétale (Tableau 1).

A un groupe, il lui est demandé d'énumérer tous les écotypes de l'espèce cultivés dans le village. La description discriminante des écotypes a été faite à partir de variables agronomiques telles que le cycle, les principales caractéristiques agromorphologiques et de variables socioéconomiques telles que l'appellation de l'écotype en langue locale mooré, la signification du nom en mooré, la provenance des semences de l'écotype. Ces séances ont été facilitées par la présentation d'échantillons de graines de chaque écotype par les producteurs agricoles détenteurs. Les semences présentées ont été collectées et conservées au Laboratoire Sol-Eau-Plante de l'INERA (Institut de l'Environnement et de Recherches Agricoles). Les producteurs ont décrit sans difficulté les différents écotypes en leur possession. Cependant, pour des raisons d'indisponibilité, les semences (graines) des cultures maraîchères, des plantes à tubercules et des espèces arboricoles n'ont pu être collectées. Les enquêtes et la collecte des semences écotypiques se sont déroulées de juillet à novembre 2010.

\section{Traitement et analyse des données}

La détermination des espèces a été faite à travers l'appui de personnes ressources 


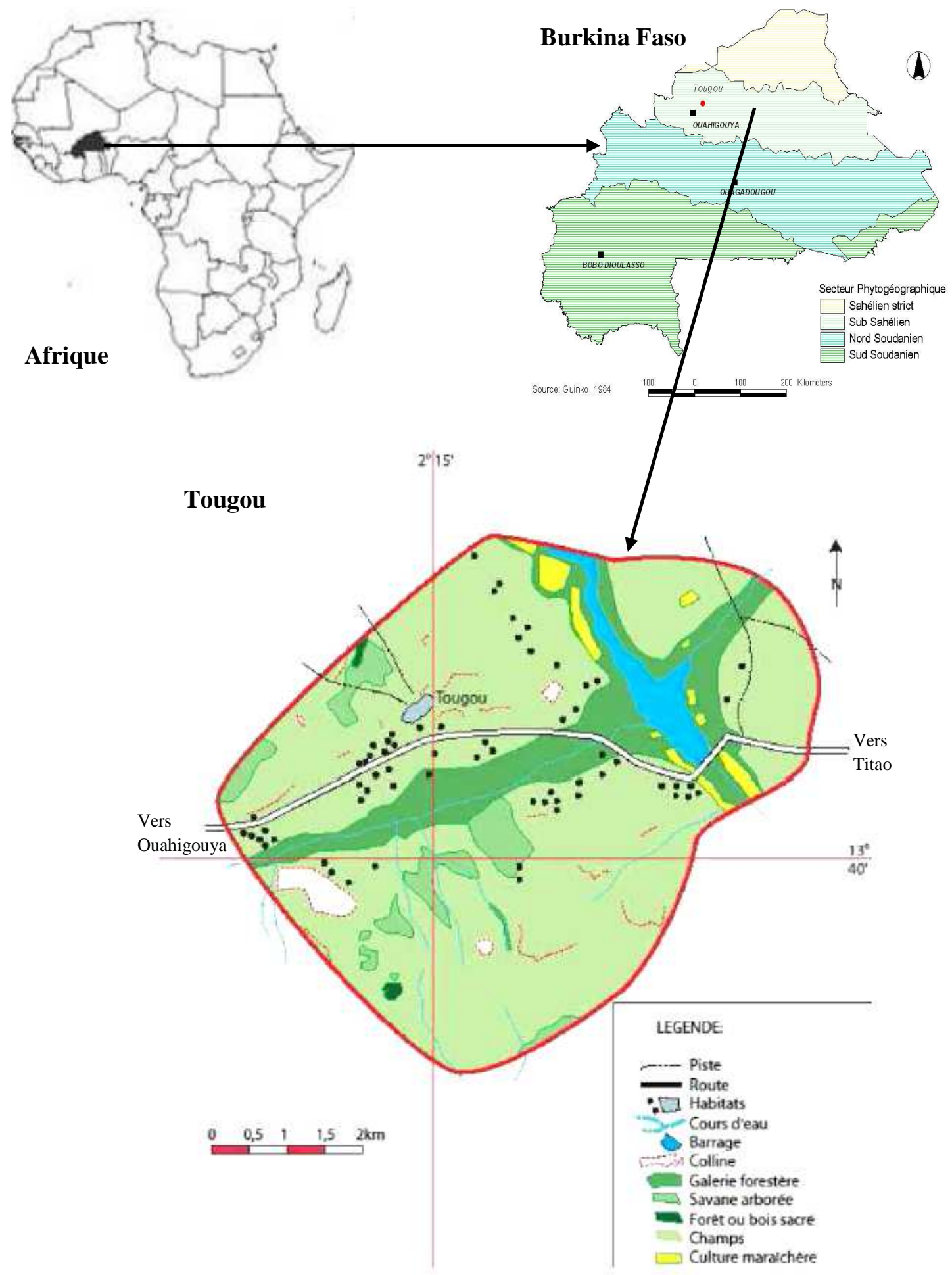

Figure 1: Localisation du site d'étude. 
Tableau 1 : Les filières considérées pour l'inventaire de la diversité écotypique.

\begin{tabular}{llccc}
\hline Groupes & Filières & \multicolumn{2}{c}{ Personnes interrogées } & Total \\
\cline { 3 - 4 } & & Hommes & Femmes & \\
\hline I & Cultures céréalières & 18 & 3 & 21 \\
II & Cultures oléo-protéagineuses & 8 & 4 & 12 \\
III & Plantes à tubercules & 10 & 1 & 11 \\
IV & Arboriculture & 5 & 0 & 5 \\
V & Cultures maraîchères de contre saison & 12 & 7 & 19 \\
VI & Cultures légumières et potagères pluviales & 2 & 5 & 7 \\
Total & & 55 & 20 & 75 \\
\hline
\end{tabular}

(Botanistes et Agronomes) et la consultation de documents tels que le catalogue des plantes vasculaires du Burkina Faso de l'IEMVT (Lebrun et al., 1991), les plantes alimentaires de l'Ouest Africain (Busson, 1965), l'action de l'homme dans la transformation de la végétation voltaïque (Guinko, 1984a). Après les enquêtes, le questionnaire a été codifié et les données d'enquêtes saisies et traitées à l'aide du logiciel SPSS 11.0. Les calculs des paramètres statistiques, notamment les proportions centésimales, ont été réalisées avec le logiciel XLSTAT-Pro 7.5.2. Les comparaisons ont été faites d'une part entre les groupes taxonomiques représentés et d'autre part entre les filières de production végétales représentées.

\section{RESULTATS}

Richesse floristique et répartition taxonomique

L'inventaire des plantes cultivées a permis de recenser 49 espèces avec 122 écotypes. Les 49 espèces se regroupaient en 39 genres et 22 familles. Leurs répartitions par familles en proportions centésimales sont consignées dans le Tableau 2.

\section{Phytodiversité par filière de production végétale \\ Diversités taxonomiques par filière de production végétale \\ Les filières maraîchère et arboricole} sont les plus diversifiées avec chacune 10 et 8 familles, 11 genres, 16 et 13 espèces, 26 et 22 écotypes respectivement. La filière des céréales qui n'est représentée que par une seule famille a comptabilisé 25 écotypes (Tableau 3).

Diversité des plantes par filière de production végétale

La filière des cultures maraîchères a présenté les espèces les plus diversifiées avec $33 \%$ de l'ensemble des espèces recensées. La filière des cultures céréalières et celle des cultures potagères pluviales ont présenté les diversités écotypiques les plus variées avec au moins deux écotypes par espèces, le sorgho ayant présenté à lui seul 10 écotypes. Les espèces ont des origines variées incluant les cinq continents (Tableau 4).

\section{Caractéristiques des écotypes Ecotypes des espèces céréalières}

Les écotypes des céréales ont des cycles compris entre $45 \mathrm{j}$ pour le mil dit "Nata" en appellation locale et $120 \mathrm{j}$ pour le sorgho localement appelé "Poug-yalmkamsé". Les écotypes de sorgho "Poug-yamlkamsé", de mil "Kibsi-Ki", de maïs "Kamaan-pelga" et de riz africain "Mouï-miougou" ayant un cycle de plus de $90 \mathrm{j}$ sont menacés de disparition. Les semences de $64 \%$ des écotypes étaient d'origine locale (Tableau 5).

\section{Ecotypes des espèces oléo protéagineuses}

Les semences de 3 écotypes sur 19 sont introduites dans le village. Les cycles des écotypes sont compris entre $40 \mathrm{j}$ pour le sésame et $90 \mathrm{j}$ pour 7 autres écotypes dont 4 (localement appelés "Beng-moaga", "Soum- 
dobgna", "Soum-wobgo", "Moa-lamdo") sont faiblement cultivés (Tableau 6).

Ecotypes des plantes à tubercules

Les 5 écotypes des plantes à tubercules étaient locaux. L'écotype de manioc appelé localement "pellé" ayant un cycle de 10 mois était en voie de disparition (Tableau 7).

\section{Ecotypes des espèces maraîchères}

Les semences de 15 écotypes sur 26 provenaient d'ailleurs. Les cycles de développement sont compris entre $30 \mathrm{j}$ pour la menthe et $110 \mathrm{j}$ pour l'écotype d'aubergine asiatique à fruit ovale et gros (Tableau 8).

\section{Ecotypes des espèces légumières et potagères pluviales}

Trois écotypes sur 25 ont été introduits. Un écotype d'oseille et 2 écotypes de calebassier ayant un cycle de plus de $90 \mathrm{j}$ sont menacés de disparition (Tableau 9).

\section{Ecotypes des espèces arboricoles}

Vingt-un écotypes sur 22 avaient un cycle de plus d'un an. Les écotypes de Azadirachta indica, de Cassia siamea et de Eucalyptus camaldulensis sont subspontanés, les semences de 13 écotypes sont importées d'autres localités (Tableau 10).

Tableau 2 : Répartition taxonomique de la flore en famille par ordre d'importance numérique de la contribution en espèces.

\begin{tabular}{lllllll}
\hline Familles & Genres & \% & Espèces & \% & Ecotypes & \% \\
\hline Solanaceae & 4 & 10,0 & 7 & 14,29 & 16 & 13,11 \\
Poaceae & 5 & 12,5 & 6 & 12,24 & 25 & 20,49 \\
Cucurbitaceae & 4 & 10,0 & 4 & 8,16 & 15 & 12,30 \\
Fabaceae & 4 & 10,0 & 4 & 8,16 & 17 & 13,93 \\
Malvaceae & 2 & 5,0 & 4 & 8,16 & 10 & 8,20 \\
Rutaceae & 1 & 2,5 & 3 & 6,12 & 4 & 3,28 \\
Anacardiaceae & 2 & 5,0 & 2 & 4,08 & 8 & 6,56 \\
Arecaceae & 2 & 5,0 & 2 & 4,08 & 2 & 1,64 \\
Convolvulaceae & 1 & 2,5 & 2 & 4,08 & 1 & 0,82 \\
Liliaceae & 1 & 2,5 & 2 & 4,08 & 2 & 1,64 \\
Myrtaceae & 2 & 5,0 & 2 & 4,08 & 3 & 2,46 \\
Caesalpiniaceae & 1 & 2,5 & 1 & 2,04 & 1 & 0,82 \\
Caricaceae & 1 & 2,5 & 1 & 2,04 & 1 & 0,82 \\
Chenopodiaceae & 1 & 2,5 & 1 & 2,04 & 2 & 1,64 \\
Composeae & 1 & 2,5 & 1 & 2,04 & 2 & 1,64 \\
Cruciferres & 1 & 2,5 & 1 & 2,04 & 3 & 2,46 \\
Euphorbiaceae & 1 & 2,5 & 1 & 2,04 & 3 & 2,46 \\
Lamiacea & 1 & 2,5 & 1 & 2,04 & 1 & 0,82 \\
Meliaceae & 1 & 2,5 & 1 & 2,04 & 1 & 0,82 \\
Musaceae & 1 & 2,5 & 1 & 2,04 & 2 & 1,64 \\
Apiaceae & 1 & 5 & 1 & 2,04 & 1 & 0,82 \\
Pedaliaceae & 1 & 2,5 & 1 & 2,04 & 2 & 1,64 \\
Total & 39 & 100 & 49 & 100 & 122 & 100 \\
\hline
\end{tabular}


Tableau 3 : Répartitions taxonomiques en proportions centésimales de la flore par filière de production végétale.

\begin{tabular}{lllllllll}
\hline Filières & Familles & \% & Genres & \% & Espèces & \% & Ecotypes & \% \\
\hline Céréales & 1 & 4 & 5 & 13 & 6 & 12 & 25 & 20 \\
Oléo protéagineux & 3 & 11 & 5 & 13 & 5 & 10 & 19 & 16 \\
Tubercules & 2 & 7 & 2 & 5 & 2 & 4 & 5 & 4 \\
Maraîchéculture & 10 & 37 & 11 & 28 & 16 & 33 & 26 & 21 \\
Potagers pluviaux & 3 & 11 & 5 & 13 & 7 & 14 & 25 & 20 \\
Arboriculture & 8 & 30 & 11 & 28 & 13 & 27 & 22 & 18 \\
Total & 27 & 100 & 39 & 100 & 49 & 100 & 122 & 100 \\
\hline
\end{tabular}

Tableau 4 : Diversité des plantes par filière de production végétale.

\begin{tabular}{|c|c|c|c|c|c|c|c|}
\hline \multirow{2}{*}{$\begin{array}{l}\text { Filières } \\
\text { végétales } \\
\text { (nombre } \\
\text { d'espèces) }\end{array}$} & \multirow{2}{*}{$\begin{array}{l}\text { Noms } \\
\text { communs }\end{array}$} & \multirow{2}{*}{$\begin{array}{l}\text { Noms } \\
\text { scientifiques }\end{array}$} & \multirow[t]{2}{*}{ Familles } & \multirow[t]{2}{*}{ Origines } & \multirow{2}{*}{$\begin{array}{l}\% \text { inter- } \\
\text { spécifiques }\end{array}$} & \multicolumn{2}{|c|}{ Ecotypes } \\
\hline & & & & & & Nombres & $\begin{array}{l}\text { \% intra- } \\
\text { spécifiques }\end{array}$ \\
\hline \multirow{6}{*}{ Céréales (6) } & Sorgho & $\begin{array}{l}\text { Sorghum } \\
\text { bicolor (L.) } \\
\text { Moench }\end{array}$ & Poaceae & Afrique & \multirow{6}{*}{12} & 10 & 40 \\
\hline & Mil & $\begin{array}{l}\text { Pennisetum } \\
\text { americanum } \\
\text { (L.) Leeke }\end{array}$ & Poaceae & Afrique & & 3 & 12 \\
\hline & Maïs & Zea mays $\mathrm{L}$. & Poaceae & Amérique & & 4 & 16 \\
\hline & Riz africain & $\begin{array}{l}\text { Oryza } \\
\text { glaberrima } \\
\text { Steud. }\end{array}$ & Poaceae & Afrique & & 4 & 16 \\
\hline & Riz asiatique & Oryza sativa $\mathrm{L}$. & Poaceae & Asie & & 2 & 8 \\
\hline & Fonio & $\begin{array}{l}\text { Digitaria exilis } \\
\text { (Kippist) Stapf }\end{array}$ & Poaceae & Afrique & & 2 & 8 \\
\hline \multirow{5}{*}{$\begin{array}{l}\text { Protéagineux } \\
\text { (5) }\end{array}$} & Niébé & $\begin{array}{l}\text { Vigna } \\
\text { unguiculata (L.) } \\
\text { Walp. }\end{array}$ & Fabaceae & Asie & \multirow{5}{*}{10} & 5 & 26 \\
\hline & Arachide & $\begin{array}{l}\text { Arachis } \\
\text { hypogea } \mathrm{L} .\end{array}$ & Fabaceae & Amérique & & 4 & 21 \\
\hline & Pois de terre & $\begin{array}{l}\text { Voandzeia } \\
\text { subterranea (L.) } \\
\text { DC. }\end{array}$ & Fabaceae & Afrique & & 7 & 37 \\
\hline & Sésame & $\begin{array}{l}\text { Sesamum } \\
\text { indicum } \mathrm{L} .\end{array}$ & Pedaliaceae & Asie & & 2 & 11 \\
\hline & Cotonnier & $\begin{array}{l}\text { Gossypium } \\
\text { hirsutum L. }\end{array}$ & Malvaceae & Amérique & & 1 & 5 \\
\hline \multirow{2}{*}{$\begin{array}{l}\text { Tubercules } \\
\text { (2) }\end{array}$} & Manioc & $\begin{array}{l}\text { Manihot } \\
\text { esculenta } \text { Crantz }\end{array}$ & Euphorbiaceae & Amérique & \multirow{2}{*}{4} & 3 & 60 \\
\hline & Patate douce & $\begin{array}{l}\text { Ipomea batatas } \\
\text { (L.) Lam. }\end{array}$ & Convolvulaceae & Amérique & & 2 & 40 \\
\hline \multirow[t]{3}{*}{$\begin{array}{l}\text { Maraîché_ } \\
\text { Culture (16) }\end{array}$} & $\begin{array}{l}\text { Aubergine } \\
\text { d'Afrique }\end{array}$ & $\begin{array}{l}\text { Solanum } \\
\text { aethiopicum L. }\end{array}$ & Solanaceae & Afrique & \multirow[t]{3}{*}{33} & 2 & 7,69 \\
\hline & $\begin{array}{l}\text { Aubergine } \\
\text { d'Asie }\end{array}$ & $\begin{array}{l}\text { Solanum } \\
\text { melongena } \mathrm{L} .\end{array}$ & Solanaceae & Asie & & 2 & 7,69 \\
\hline & Tomate & $\begin{array}{l}\text { Lycopersicum } \\
\text { esculentum Mill. }\end{array}$ & Solanaceae & Amérique & & 3 & 11,54 \\
\hline
\end{tabular}


D. BAMBARA et al. / Int. J. Biol. Chem. Sci. 5(6): 2415-2433, 2011

\begin{tabular}{|c|c|c|c|c|c|c|c|}
\hline & Oignon & Allium cepa $\mathrm{L}$. & Liliaceae & Asie & & 1 & 3,85 \\
\hline & Piment & Capsicum & Solanaceae & Amérique & & 3 & 11,54 \\
\hline & Concombre & $\begin{array}{l}\text { frutescens } \mathrm{L} . \\
\text { Cucumis sativus } \\
\text { L. }\end{array}$ & Cucurbitaceae & Afrique & & 1 & 3,85 \\
\hline & Ail & $\begin{array}{l}\text { Allium sativum } \\
\text { L. }\end{array}$ & Liliaceae & Asie & & 1 & 3,85 \\
\hline & Poivron & $\begin{array}{l}\text { Capsicum } \\
\text { annum } \mathrm{L} .\end{array}$ & Solanaceae & Amérique & & 1 & 3,85 \\
\hline & Carotte & $\begin{array}{l}\text { Daucus carota } \\
\text { L. }\end{array}$ & Apiaceae & Asie & & 1 & 3,85 \\
\hline & Epinard & $\begin{array}{l}\text { Spinacia } \\
\text { oleracea } \mathrm{L} .\end{array}$ & Chenopodiaceae & Asie & & 2 & 7,69 \\
\hline & Chou & $\begin{array}{l}\text { Brassica } \\
\text { oleracea } \mathrm{L} .\end{array}$ & Crucifères & Europe & & 2 & 7,69 \\
\hline & Laitue & $\begin{array}{l}\text { Lactuca sativa } \\
\text { L. }\end{array}$ & Composeae & Asie & & 2 & 7,69 \\
\hline & $\begin{array}{l}\text { Pomme de } \\
\text { terre }\end{array}$ & $\begin{array}{l}\text { Solanum } \\
\text { tuberosum L. }\end{array}$ & Solanaceae & Amérique & & 2 & 7,69 \\
\hline & Haricot vert & $\begin{array}{l}\text { Phaseolus } \\
\text { vulgaris } \mathrm{L} .\end{array}$ & Fabaceae & Amérique & & 1 & 3,85 \\
\hline & Menthe & $\begin{array}{l}\text { Mentha piperita } \\
\text { L. }\end{array}$ & Lamiaceae & Europe & & 1 & 3,85 \\
\hline & Persil & $\begin{array}{l}\text { Petroselinum } \\
\text { sativum Hoffm. }\end{array}$ & Apiaceae & Europe & & 1 & 3,85 \\
\hline \multirow{7}{*}{$\begin{array}{l}\text { Potagers } \\
\text { pluviaux } \\
\text { (7) }\end{array}$} & Pastèque & $\begin{array}{l}\text { Citrullus } \\
\text { vulgaris } \mathrm{L} .\end{array}$ & Cucurbitaceae & Afrique & \multirow{7}{*}{14} & 5 & 20 \\
\hline & Oseille-Bissap & $\begin{array}{l}\text { Hibiscus } \\
\text { sabdariffa } \mathrm{L} .\end{array}$ & Malvaceae & Asie & & 4 & 16 \\
\hline & Oseille-fibre & $\begin{array}{l}\text { Hibiscus } \\
\text { cannabinus L. }\end{array}$ & Malvaceae & Afrique & & 2 & 8 \\
\hline & Calebassier & $\begin{array}{l}\text { Lagenaria } \\
\text { siceraria (Mol.) } \\
\text { Standl. }\end{array}$ & Cucurbitaceae & Afrique & & 5 & 20 \\
\hline & Gombo & $\begin{array}{l}\text { Hibiscus } \\
\text { esculentus L. }\end{array}$ & Malvaceae & Afrique & & 3 & 12 \\
\hline & Tabac & $\begin{array}{l}\text { Nicotiana } \\
\text { tabacum L. }\end{array}$ & Solanaceae & Amérique & & 2 & 8 \\
\hline & Courge & $\begin{array}{l}\text { Cucurbita pepo } \\
\text { L. }\end{array}$ & Cucurbitaceae & Amérique & & 4 & 16 \\
\hline \multirow{9}{*}{$\begin{array}{l}\text { Arbori- } \\
\text { Culture } \\
\text { (13) }\end{array}$} & Manguier & $\begin{array}{l}\text { Mangifera indica } \\
\text { L. }\end{array}$ & Anacardiaceae & Asie & \multirow[t]{9}{*}{27} & 5 & 22,73 \\
\hline & Goyavier & $\begin{array}{l}\text { Psidium guajava } \\
\text { L. }\end{array}$ & Myrtaceae & Amérique & & 2 & 9,09 \\
\hline & Oranger & $\begin{array}{l}\text { Citrus aurantium } \\
\text { L. }\end{array}$ & Rutaceae & Asie & & 1 & 4,55 \\
\hline & Citronnier & $\begin{array}{l}\text { Citrus } \\
\text { aurantifolia } \\
\text { Swingle }\end{array}$ & Rutaceae & Asie & & 2 & 9,09 \\
\hline & Bananier & $\begin{array}{l}\text { Musa } \\
\text { sapienthum } \mathrm{L} .\end{array}$ & Musaceae & Asie & & 2 & 9,09 \\
\hline & Papayer & $\begin{array}{l}\text { Carica papaya } \\
\text { L. }\end{array}$ & Caricaceae & Amérique & & 1 & 4,55 \\
\hline & Anacardier & $\begin{array}{l}\text { Anacardium } \\
\text { occidentale } \mathrm{L} .\end{array}$ & Anacardiaceae & Amérique & & 3 & 13,64 \\
\hline & Mandarinier & $\begin{array}{l}\text { Citrus nobilis } \\
\text { Lour. }\end{array}$ & Rutaceae & Asie & & 1 & 4,55 \\
\hline & Palmier à huile & $\begin{array}{l}\text { Elaeis guineensis } \\
\text { Jacq. }\end{array}$ & Arecaceae & Afrique & & 1 & 4,55 \\
\hline
\end{tabular}




\begin{tabular}{|c|c|c|c|c|c|}
\hline Palmier dattier & $\begin{array}{l}\text { Phoenix } \\
\text { dactylifera L. }\end{array}$ & Arecaceae & Asie & 1 & 4,55 \\
\hline Neem & $\begin{array}{l}\text { Azadirachta } \\
\text { indica A. Juss. }\end{array}$ & Meliaceae & Asie & 1 & 4,55 \\
\hline Cassia & $\begin{array}{l}\text { Cassia siamea } \\
\text { Lam. }\end{array}$ & Caesalpiniaceae & Asie & 1 & 4,55 \\
\hline Eucalyptus & $\begin{array}{l}\text { Eucalyptus } \\
\text { camaldulensis } \\
\text { Dehnardt }\end{array}$ & Myrtaceae & Océanie & 1 & 4,55 \\
\hline
\end{tabular}

Tableau 5 : Caractéristiques écotypiques des espèces céréalières.

\begin{tabular}{|c|c|c|c|c|c|}
\hline $\begin{array}{l}\text { Espèces et } \\
\text { numéro de } \\
\text { l'écotype }\end{array}$ & $\begin{array}{l}\text { Appellation } \\
\text { locale de } \\
\text { l'écotype } \\
\end{array}$ & $\begin{array}{l}\text { Signification } \\
\text { du nom }\end{array}$ & $\begin{array}{l}\text { Cycles } \\
\text { (jours) }\end{array}$ & $\begin{array}{l}\text { Provenances } \\
\text { des semences }\end{array}$ & $\begin{array}{l}\text { Principales } \\
\text { caractéristiques agro- } \\
\text { morphologiques }\end{array}$ \\
\hline \multicolumn{6}{|l|}{$\underline{\text { Sorgho }}$} \\
\hline 1 & Kapelga & Sorgho blanc & 70 & Locale & Glume grise \\
\hline 2 & Gnamnè & Sorgho noir & 80 & Locale & Glume noire \\
\hline 3 & Kazèga & Sorgho rouge & 80 & Locale & Grain rouge \\
\hline 4 & Loumbo & Sorgho à grain dur & 70 & INERA & Gros grain, panicule \\
\hline 5 & Kon- & Chèvre non & 50 & Locale & compacte \\
\hline 6 & Kossibouga & vendue & 50 & Locale & Taille relativement petite \\
\hline 7 & Balinga & Sorgho ordinaire & 100 & Locale & Grain très petit \\
\hline 8 & Kazonga* & Sorgho aveugle & 90 & Locale & Bas-fonds, glume fermée \\
\hline 9 & Maca & Sorgho-miel & 120 & Locale & Grain sucré \\
\hline 10 & $\begin{array}{l}\text { Poug- } \\
\text { yamlkamsé* } \\
\text { Lobinn-ko }\end{array}$ & $\begin{array}{l}\text { Bras de femme } \\
\text { idiote } \\
\text { Semis en volée }\end{array}$ & 50 & Locale & $\begin{array}{l}\text { Longue panicule }(50 \mathrm{~cm}) \\
\text { Grain rouge, tombe à } \\
\text { maturité }\end{array}$ \\
\hline \multicolumn{6}{|r|}{ 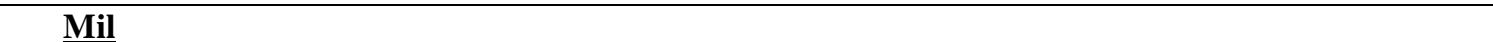 } \\
\hline 1 & Balbou & Mil noir & 80 & Pobé Mengao & Epi de $30 \mathrm{~cm}$, graine \\
\hline 2 & Nata & Mil court & 45 & Sahel (Mali) & brune \\
\hline 3 & Kibsi-Ki* & Mil des Dogon & 90 & $\begin{array}{l}\text { Pays Dogon } \\
\text { (Mali) }\end{array}$ & $\begin{array}{l}\text { Taille courte, petit épi } \\
\text { Epi plus long que Balbou }\end{array}$ \\
\hline \multicolumn{6}{|r|}{ 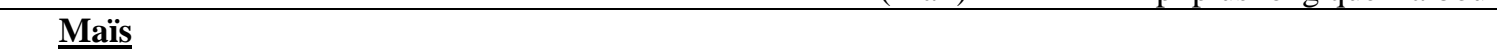 } \\
\hline$\overline{1}$ & Yakka-raguin & Voisin ne dort pas & 65 & Locale & Un seul épi costaud \\
\hline 2 & Kamaan- & Maïs femelle & 90 & Locale & Deux épis valides \\
\hline 3 & gnanga & Maïs blanc & 100 & INERA & Grain blanc (SR21) \\
\hline 4 & $\begin{array}{l}\text { Kamaan-pelga* } \\
\text { Koobo }\end{array}$ & Agriculture & 70 & INERA & Grain rouge \\
\hline \multicolumn{6}{|l|}{ Riz africain } \\
\hline 1 & Koud-poko & Hâtif & 60 & Locale & Grain court et gros \\
\hline 2 & Mouï-kenda & Panicule pendante & 70 & Locale & Long grain \\
\hline 3 & Mouï-Nadenga & Riz sauvage & 70 & Locale & Chute du grain à maturité \\
\hline 4 & $\begin{array}{l}\text { Mouï- } \\
\text { miougou* }\end{array}$ & Riz rouge & 90 & Locale & Grain marron \\
\hline \multicolumn{6}{|l|}{ Riz asiatique } \\
\hline 1 & Mouï-Kollé & Grain long & 90 & INERA & Grain blanc, taille $(1,5 \mathrm{~m})$ \\
\hline 2 & Chinoir & Riz chinois & 90 & INERA & Grain tout blanc \\
\hline \multicolumn{6}{|l|}{ Fonio } \\
\hline 1 & Kiou-belké & Fonio amélioré & 90 & INERA & Gros grain \\
\hline 2 & Kiou-zalga & Fonio simple & 90 & Locale & Petit grain \\
\hline
\end{tabular}


Tableau 6 : Caractéristiques écotypiques des espèces oléo protéagineuses.

\begin{tabular}{|c|c|c|c|c|c|}
\hline $\begin{array}{l}\text { Espèces et } \\
\text { numéro de } \\
\text { l'écotype }\end{array}$ & $\begin{array}{l}\text { Appellation } \\
\text { locale de } \\
\text { l'écotype }\end{array}$ & $\begin{array}{l}\text { Signification } \\
\text { du nom }\end{array}$ & $\begin{array}{l}\text { Cycles } \\
\text { (jours) }\end{array}$ & $\begin{array}{l}\text { Provenances } \\
\text { des } \\
\text { semences }\end{array}$ & $\begin{array}{l}\text { Principales } \\
\text { caractéristiques agro- } \\
\text { morphologiques }\end{array}$ \\
\hline \multicolumn{6}{|l|}{ Niébé } \\
\hline 1 & Beng-raaga & Niébé mâle & 50 & Locale & Port rampant \\
\hline 2 & Kon-diksi- & Pas de récolte la & 70 & Locale & Gousse et grains noirs \\
\hline 3 & gnoungou & nuit & 90 & Locale & Port très rampant \\
\hline 4 & Beng-moaga* & Niébé mossi & 45 & Locale & Port peu rampant \\
\hline 5 & $\begin{array}{l}\text { Kom-kallé } \\
\text { Beng-siidou }\end{array}$ & $\begin{array}{l}\text { Combat la } \\
\text { soudure } \\
\text { Niébé sucré }\end{array}$ & 70 & Gorom & Grain rouge \\
\hline \multicolumn{6}{|l|}{ Arachide } \\
\hline 1 & Nassar-souma & Arachide du & 50 & INERA & Variété améliorée \\
\hline 2 & Soum-pelga & Blanc & 50 & Locale & (TE3) \\
\hline 3 & Soum-miougou & Arachide blanche & 50 & Locale & Graine blanche \\
\hline 4 & Soum-dobgna* & $\begin{array}{l}\text { Arachide rouge } \\
\text { Arachide érigée }\end{array}$ & 90 & Locale & $\begin{array}{l}\text { Graine rouge } \\
+/=3 \text { graines/gousse }\end{array}$ \\
\hline \multicolumn{6}{|l|}{ Pois de terre } \\
\hline 1 & Poko & Pour les femmes & 50 & Locale & Grain blanc \\
\hline 2 & Kon-Kin- & Dehors coépouse & 50 & Locale & Grain bigarré \\
\hline 3 & nipougto & Eléphant & 90 & Locale & Gros grain rouge \\
\hline 4 & Soum-wobgo* & Perdrix & 90 & Locale & Grain couleur perdrix \\
\hline 5 & Soum-koadinnga & Tourterelle & 90 & Locale & Grain couleur \\
\hline 6 & Soum-wallé & Rouge & 90 & Locale & tourterelle \\
\hline 7 & $\begin{array}{l}\text { Soum-miougou } \\
\text { Soum-saballé }\end{array}$ & Noir & 60 & Locale & $\begin{array}{l}\text { Grain petit, rouge } \\
\text { Grain noir }\end{array}$ \\
\hline \multicolumn{6}{|l|}{ Sésame } \\
\hline 1 & Sinn-pelga & Sésame blanc & 40 & INERA & Grain blanc (S42) \\
\hline 2 & Sinn-miougou & Sésame rouge & 40 & Locale & Grain bigarré \\
\hline \multicolumn{6}{|l|}{ Cotonnier } \\
\hline 1 & Moa-lamdo* & Cotonnier mossi & 90 & Locale & Tige rouge foncé \\
\hline
\end{tabular}


Tableau 7 : Caractéristiques écotypiques des plantes à tubercules.

\begin{tabular}{|c|c|c|c|c|c|}
\hline $\begin{array}{l}\text { Espèces et } \\
\text { numéro de } \\
\text { l'écotype }\end{array}$ & $\begin{array}{l}\text { Appellation } \\
\text { locale } \\
\text { de l'écotype }\end{array}$ & $\begin{array}{l}\text { Signification } \\
\text { du nom }\end{array}$ & Cycles & $\begin{array}{l}\text { Provenances } \\
\text { des semences }\end{array}$ & $\begin{array}{l}\text { Principales } \\
\text { caractéristiques agro- } \\
\text { morphologiques }\end{array}$ \\
\hline \multicolumn{6}{|l|}{ Manioc } \\
\hline 1 & Pellé* & Manioc & 10 mois & Locale & Pétiole blanc \\
\hline 2 & Miougou & blanc & 6 mois & Locale & Pétiole rouge, limbes \\
\hline 3 & $\begin{array}{l}\text { Bandakou- } \\
\text { soukarê }\end{array}$ & $\begin{array}{l}\text { Manioc } \\
\text { rouge } \\
\text { Manioc } \\
\text { sucré }\end{array}$ & 6 mois & Locale & $\begin{array}{l}\text { pentadigités } \\
\text { Tubercule sucré, limbes } \\
\text { septadigités }\end{array}$ \\
\hline \multicolumn{6}{|l|}{ Patate douce } \\
\hline 1 & Nagnoui-pellé & Patate & 90 jours & Locale & Tubercule à chair blanche \\
\hline 2 & $\begin{array}{l}\text { Nagnoui- } \\
\text { miougou }\end{array}$ & $\begin{array}{l}\text { blanche } \\
\text { Patate rouge }\end{array}$ & 90 jours & Locale & $\begin{array}{l}\text { Tubercule à chair } \\
\text { rougeâtre }\end{array}$ \\
\hline
\end{tabular}

* Ecotypes en voie de disparition

Tableau 8 : Caractéristiques écotypiques des cultures maraîchères de contre saison.

\begin{tabular}{|c|c|c|c|c|c|}
\hline $\begin{array}{l}\text { Espèces et } \\
\text { numéro de } \\
\text { l'écotype }\end{array}$ & $\begin{array}{l}\text { Appellation } \\
\text { locale de } \\
\text { l'écotype }\end{array}$ & $\begin{array}{l}\text { Signification } \\
\text { du nom }\end{array}$ & $\begin{array}{l}\text { Cycles } \\
\text { (jours) }\end{array}$ & $\begin{array}{l}\text { Provenances } \\
\text { des } \\
\text { semences }\end{array}$ & $\begin{array}{l}\text { Principales } \\
\text { caractéristiques agro- } \\
\text { morphologiques }\end{array}$ \\
\hline \multicolumn{6}{|l|}{$\underline{\text { Aubergine }}$} \\
\hline \multicolumn{6}{|l|}{$\underline{\text { africaine }}$} \\
\hline 1 & Koumb-zallé & Aubergine simple & 60 & Locale & Fruit arrondi, vert foncé \\
\hline 2 & Bid-koumba & Aubergine de Bidi & 60 & Bidi & $\begin{array}{l}\text { Fruit arrondi plus gros, } \\
\text { blanc }\end{array}$ \\
\hline \multicolumn{6}{|l|}{ Aubergine } \\
\hline 1 & Guilsi & Rond & 90 & Ouahigouya & Fruit rond \\
\hline 2 & Silinga & Allongé & 110 & Ouahigouya & Fruit ovale, plus gros \\
\hline \multicolumn{6}{|l|}{ Tomate } \\
\hline 1 & Tolma & Long & 90 & INERA & Fruit allongé \\
\hline 2 & Rome & Vient de Rome & 90 & Ghana & Fruit rouge, rond ne pourrit \\
\hline 3 & Guilsi & Rond & 90 & Locale & $\begin{array}{l}\text { pas } \\
\text { Fruit arrondi, rouge }\end{array}$ \\
\hline \multicolumn{6}{|l|}{$\underline{\text { Oignon }}$} \\
\hline 1 & $\begin{array}{l}\text { Gabd- } \\
\text { miougou }\end{array}$ & Oignon rouge & 90 & Ouahigouya & Bulbe rouge \\
\hline
\end{tabular}




\begin{tabular}{clllll}
\hline Piment & & & & \\
1 & Soubaga & Sorcier (en & 60 & Locale & Fruit rouge, très piquant \\
2 & Liouli-kiparé & Dioula) & 60 & Locale & Petit fruit rouge \\
3 & Kipar- & Piment des & 60 & Locale & Gros fruit non piquant \\
& broudou & oiseaux & & & \\
& & Gros & & & \\
\hline
\end{tabular}

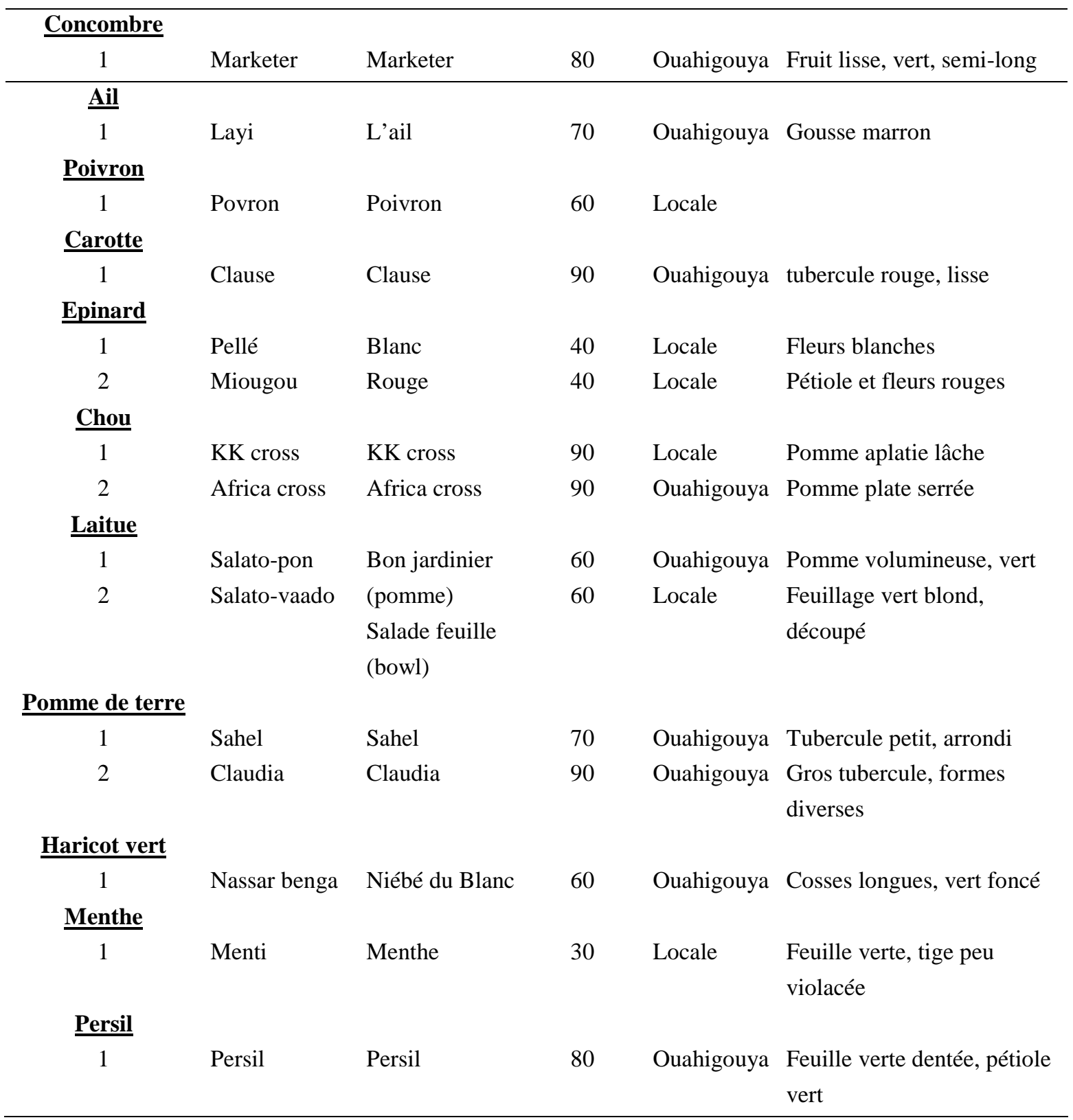


Tableau 9: Caractéristiques écotypiques des cultures légumières et potagères pluviales.

\begin{tabular}{|c|c|c|c|c|c|}
\hline $\begin{array}{c}\text { Espèces et } \\
\text { numéro de } \\
\text { l'écotype }\end{array}$ & $\begin{array}{l}\text { Appellation } \\
\text { locale de } \\
\text { l'écotype }\end{array}$ & $\begin{array}{l}\text { Signification } \\
\text { du nom }\end{array}$ & $\begin{array}{l}\text { Cycles } \\
\text { (jours) }\end{array}$ & $\begin{array}{l}\text { Provenances } \\
\text { des } \\
\text { semences }\end{array}$ & $\begin{array}{l}\text { Principales } \\
\text { caractéristiques agro } \\
\text { morphologiques }\end{array}$ \\
\hline \multicolumn{6}{|l|}{ Pastèque } \\
\hline 1 & Pellé & Peau vert clair & 70 & Locale & Peau vert clair \\
\hline 2 & Miougou & Rouge & 70 & Locale & Chair rouge foncé \\
\hline 3 & Saballé & Noire & 70 & Locale & Peau noire \\
\hline 4 & Goulounga & Peau rayée & 70 & Locale & Peau rayée vert-vert \\
\hline \multirow[t]{2}{*}{5} & Roumana & Peau jaune & 60 & Locale & foncé \\
\hline & & & & & Peau jaunâtre \\
\hline \multicolumn{6}{|l|}{ Oseille-bissap } \\
\hline 1 & Bi-miougou & Oseille rouge & 80 & Locale & Tige noire, nervure \\
\hline 2 & Bi-pellé & Oseille blanche & 80 & Locale & rouge \\
\hline 3 & Sa-touuma* & Murit en fin de & 100 & Locale & Feuille et calice blancs \\
\hline 4 & Wegda & saison & 90 & Locale & Fruit plus gros \\
\hline \multirow[t]{3}{*}{5} & Bissap & Oseille à fruit & 90 & Locale & Calice plus long, sauce \\
\hline & & allongé & & & Calice rouge foncé \\
\hline & & «Bissap » & & & \\
\hline \multicolumn{6}{|l|}{ Oseille-fibre } \\
\hline 1 & Bêrinsé- & Rouge & 60 & Locale & Tiges et pétioles \\
\hline \multirow[t]{2}{*}{2} & miougou & Noir & 60 & Locale & rouges \\
\hline & Bêrinsé-Saballé & & & & Tiges et pétioles verts \\
\hline \multicolumn{6}{|l|}{ Calebassier } \\
\hline 1 & Rakkèk-ma & Calebasse & 90 & Locale & Fruit moyen \\
\hline 2 & Wam-Bêda* & moyenne & 120 & Locale & Très gros fruit \\
\hline 3 & Wam-gnaba & Grosse calebasse & 90 & Locale & Fruit avec des boutons \\
\hline 4 & Linnga* & Peau - bouton & 110 & Locale & Gourde de formes \\
\hline \multirow[t]{2}{*}{5} & Souutissi & Gourde & 90 & Locale & diverses \\
\hline & & Louche & & & $\begin{array}{l}\text { Louche de tailles } \\
\text { diverses }\end{array}$ \\
\hline \multicolumn{6}{|l|}{$\underline{\text { Gombo }}$} \\
\hline 1 & Gnaga-yiila & Corne d'antilope & 60 & Locale & Fruit en corne \\
\hline 2 & Maan-saballé & Gombo noir & 60 & Locale & d'antilope \\
\hline \multirow[t]{2}{*}{3} & Maan-miougou & Gombo rouge & 60 & Locale & Fruit de couleur noire \\
\hline & & & & & Fruit de couleur rouge \\
\hline \multicolumn{6}{|l|}{$\underline{\text { Tabac }}$} \\
\hline 1 & Tab-moaga & Tabac des Mossi & 90 & Locale & Hauteur atteignant 40 \\
\hline \multirow[t]{2}{*}{2} & Nassar-taba & Tabac du Blanc & 90 & Bobo & $\mathrm{cm}$ \\
\hline & & & & & Hauteur atteignant $1 \mathrm{~m}$ \\
\hline
\end{tabular}




\section{Courge}

$\begin{array}{llllll}1 & \text { Yog-pellé } & \text { Courge blanche } & 90 & \text { Locale } & \text { Fruit ovale } \\ 2 & \text { Yog-miougou } & \text { Courge rouge } & 90 & \text { Locale } & \text { Fruit arrondi } \\ 3 & \text { Maï-maana } & \text { Gombo des Maïga } & 90 & \text { Mali } & \text { Fruit ovale plus } \\ 4 & \text { Niri } & \text { Courge jaune } & 50 & \text { Ouahigouya } & \text { allongé } \\ & & & & \begin{array}{l}\text { Fruit jaune à chair } \\ \text { sucrée }\end{array}\end{array}$

* Ecotypes en voie de disparition

Tableau 10 : Caractéristiques écotypiques des espèces arboricoles.

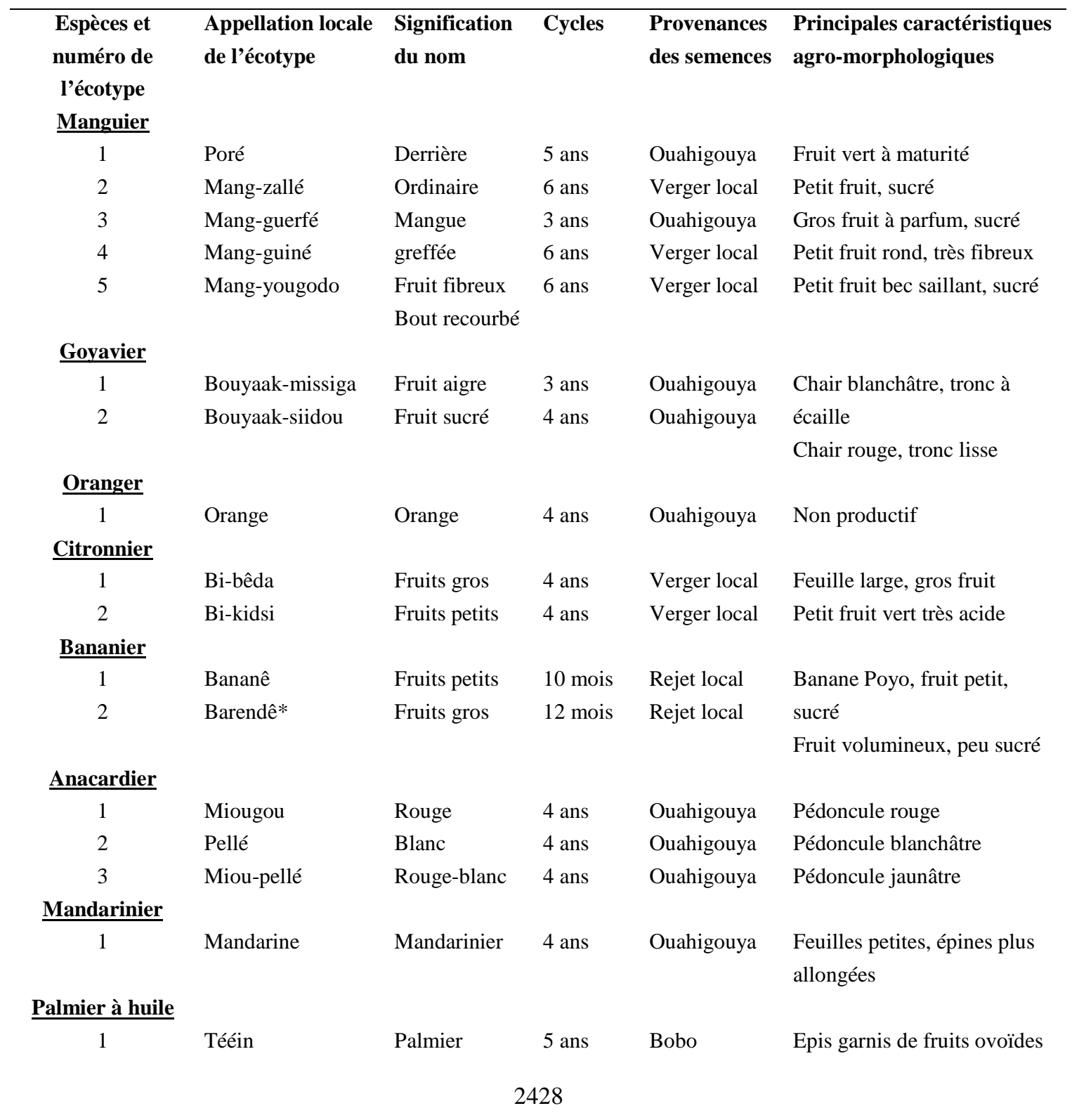




\begin{tabular}{clllll}
\hline $\begin{array}{c}\text { Palmier dattier } \\
1\end{array}$ & Datte & Dattier & 5 ans & Mali & $\begin{array}{l}\text { jaune-noir } \\
\text { Tronc cylindrique, feuille } \\
\text { composée pennée }\end{array}$ \\
$\frac{\text { Neem }}{1}$ & Nima & Neem & 4 & Subspontané & $\begin{array}{l}\text { Ecorce très marquée, feuille } \\
\text { mince }\end{array}$ \\
\hline$\frac{\text { Cassia }}{1}$ & Cassia & Cassia & 5 & Subspontané & $\begin{array}{l}\text { Feuille vert foncé, branches } \\
\text { blanchâtres }\end{array}$ \\
\hline Eucalyptus & Eucalyptus & Eucalyptus & 4 & Ouahigouya & $\begin{array}{l}\text { Tronc lisse, blanchâtre, très } \\
\text { haut }\end{array}$ \\
\hline Ecotypes en voie de disparition & & &
\end{tabular}

* Ecotypes en voie de disparition

\section{DISCUSSION}

Les résultats de l'inventaire révèlent la diversité des cultures maintenues par les producteurs de Tougou avec 49 espèces représentant $73 \%$ des espèces cultivées au plan national (Balma et al., 2004 ; CAPES, 2006). Tougou abrite ainsi une grande partie des plantes cultivées du Burkina Faso. Des espèces recensées, les plus représentées sont les Solanaceae, les Poaceae et les Cucurbitaceae avec 14,29\%, les Fabaceae avec $12,24 \%$ et les Malvaceae avec $8,16 \%$. Les Caesalpiniaceae, les Caricaceae, les Chenopodiaceae, les Composeae, les Crucifères, les Euphorbiaceae, les Lamiacea, les Meliaceae, les Musaceae, les Apiadaceae et les Pedaliaceae, soit 11 familles, ne sont représentées que par une seule espèce et un seul genre. A notre connaissance, aucun inventaire systématique antérieur n'a été effectué sur les plantes cultivées du Burkina Faso. La plupart des travaux d'inventaire sont réalisés par filière de production végétale compte tenu des objectifs et de la spécificité de ces espèces cultivées. Néanmoins, nos résultats taxonomiques corroborent en partie ceux de Traoré (1991) qui a trouvé que la flore adventice des céréales du Burkina Faso est dominée par les Poaceae et les Fabaceae.
La diversité interspécifique de la filière des cultures maraîchères représente $33 \%$ de l'ensemble des espèces recensées contre $27 \%$ pour la filière arboricole, $14 \%$ pour les potagers pluviaux, $12 \%$ pour les céréales, $10 \%$ pour les oléo-protéagineux et $4 \%$ pour les plantes à tubercules. Les plantes cultivées déjà recensées au Burkina Faso par d'autres chercheurs (Lebrun et al., 1991 ; Balma et al., 2004) sont dominées par 27 espèces maraîchères, 12 espèces arboricoles, 7 espèces à tubercules et 6 espèces céréalières. Nos observations sont conformes à ces résultats à l'exception des plantes à tubercules parmi lesquelles 2 espèces seulement sont recensées. Ainsi, le village de Tougou devient une zone de la conservation de la biodiversité des plantes cultivées du Burkina Faso. En effet, nos résultats, comparés à ceux obtenus au niveau national (Balma et al., 2004 ; CAPES, 2006) révèlent la présence dans le village de $100 \%$ des espèces de céréales, de $29 \%$ des plantes à tubercules, de $56 \%$ des espèces oléoprotéagineuses, de $82 \%$ des espèces de cultures maraîchères légumières et potagères, et de $83 \%$ des espèces arboricoles. Du point de vue de la diversité écotypique, nos résultats indiquent que le sorgho (Sorghum bicolor (L.) Moench), le pois de terre (Voandzeia 
sbterranea (L.) DC.), le niébé (Vigna unguiculata (L.) Walp.), la pastèque (Citrillus vulgaris L.), le calebassier (Lagenaria sicerraria (Mol.) Standl.) et le manguier (Manguifera indica L.) présentent chacun au moins 5 écotypes. Toutes ces espèces à l'exception du niébé et du manguier sont originaires d'Afrique (Guinko, 1984a). Les autres espèces cultivées présentent moins de 5 écotypes par espèce.

La diversité intra-spécifique des plantes cultivées est nettement en deçà du potentiel national qui compte 870 écotypes de sorgho, 333 écotypes de mil, 201 écotypes de maïs, 527 écotypes de riz, 161 écotypes de niébé, 59 écotypes de voandzou, 6 écotypes de patate (Sié et al., 1998; CAPES, 2006). Cette faiblesse de la diversité intra-spécifique peut s'expliquer en partie par la péjoration climatique qui ne garantit pas le développement normal des espèces (INRAFAO, 2006). Au total, 5 écotypes de céréales, 4 écotypes des plantes oléo protéagineuses, 3 écotypes des plantes légumières et potagères pluviales et un écotype des plantes à tubercules, sont 13 des écotypes appartenant à 12 espèces regroupées dans 10 genres et 6 familles menacés de disparition. Ces écotypes menacés qui ont un cycle de plus de $90 \mathrm{j}$ représentent $10,5 \%$ de l'ensemble des écotypes recensés. Le contexte pluviométrique actuel rend les producteurs septiques à leur adoption, ce qui entraîne une perte de la diversité biologique (Sié et al., 1998). Ce constat a été fait par Naeem et Defries (2009) qui attribuent cette menace de perte de diversité biologique au changement climatique. Aussi, l'absence d'une diversité des écosystèmes liée à leur dégradation (FIDA, 2008; Mbayngone et al., 2008; Ouédraogo, 2009) et la relative homogénéité des conditions écologiques à l'échelle du village (Ouédraogo, 2009), sont des facteurs défavorables à l'existence d'une diversité d'espèces. La faible diversité intra-spécifique observée dans le village Tougou rend les espèces végétales plus exposées à l'extinction. En effet, c'est la diversité génétique qui permet la pérennisation des espèces (CBD, 2009).

Les producteurs réagissent à la perte de la diversité écotypique en important des semences d'écotypes originaires d'autres localités. Sur les 122 écotypes recensés, 79 sont locaux tandis que les semences des 43 autres écotypes sont importées de localités diverses. La dénomination des écotypes en langue locale, la connaissance des cycles et la description des caractères agromorphologiques essentiels sont autant d'éléments qui prouvent que les producteurs ont une bonne connaissance de la diversité des écotypes qu'ils cultivent. D'ailleurs, les espèces végétales cultivées à Tougou sont composées de $27 \%$ d'espèces africaines et de $73 \%$ d'espèces provenant des 4 autres continents. Cette observation est semblable à celle de Guinko (1984a) qui a signalé que la flore cultivée au Burkina Faso est dominée par des espèces allochtones qui représentent $88 \%$ contre $12 \%$ pour les espèces autochtones. La péjoration climatique et son corollaire de dégradation des écosystèmes joueraient en défaveur des espèces étrangères qui n'arrivent plus à s'adapter au milieu expliquant ainsi la baisse de leur représentativité indiquée par nos résultats obtenus en 2010 comparativement aux années 1984.

\section{Conclusion}

La diversité interspécifique est élevée dans le village Tougou au Nord du Burkina Faso. En revanche, la diversité intraspécifique est très faible menaçant ainsi la survie des espèces. L'étude montre que Tougou renferme la plupart des espèces végétales cultivées au Burkina Faso. Les espèces de la famille des Solanaceae sont les 
plus nombreuses. L'influence du changement climatique se traduit par une perte de la phytodiversité des deux façons suivantes: (i) abandon des espèces et écotypes dont les cycles de développement sont longs; (ii) disparition des espèces et écotypes pour cause de dégradation des agrosystèmes où ils sont cultivés. Ainsi, 10,5\% des écotypes sont identifiés comme menacés de disparition. Des actions telles que les pratiques traditionnelles de la culture associée, la promotion de la pénétration des variétés améliorées adaptées, la restauration des écosystèmes dégradés, etc., doivent être entreprises de concert avec les populations en vue de promouvoir dans leur terroir les espèces et écotypes menacés ayant un intérêt économique et social. Une stratégie de collecte, de conservation et de promotion de ces ressources végétales s'avère nécessaire. Pour mieux préciser la diversité des cultures dans le village, des études approfondies incluant la caractérisation des écotypes par des expérimentations sur le terrain et par voie moléculaire doivent être réalisées.

De telles études doivent être menées dans tous les villages du pays car, avec la diversité ethnique du Burkina Faso (plus de 60 ethnies), l'identification d'écotypes nouveaux intéressants est probable et peut être exploitée pour de meilleures pratiques d'adaptation au changement climatique. Cela sous-entend que les populations rurales disposent de connaissances endogènes qui devront être renforcées et vulgarisées.

\section{REMERCIEMENTS}

Nous remercions le projet RIPIECSA (Recherche Interdisciplinaire et Participative sur les Interactions entre les Écosystèmes, le Climat et les Sociétés d'Afrique de l'Ouest) qui a financé cette étude. Nous disons merci à Dr Amadé Ouédraogo, Maître assistant en Botanique et Phytoécologie à l'Université de Ouagadougou, à M. François W. Kagambèga et M. Philippe Bayen, doctorants au Laboratoire de Biologie et Ecologie Végétales de l'UFR/SVT de l'Université de Ouagadougou pour les observations et critiques apportées au manuscrit.

\section{REFERENCES}

Baco MN, Ahanchede A, Bello S, Dansi A, Vodouhe R, Biaou G, Lescure J-P. 2008. Évaluation des pratiques de gestion de la diversité du niébé (Vigna unguiculata): une tentative méthodologique expérimentée au Bénin. Cah. Agric,. 17(2): 183-188.

Balma D, Bognounou O, Ouédraogo A, Tankoano MJ, Zigani G, Zigani M. 2004. La diversité biologique agricole au Burkina Faso. In La Biodiversité Agricole en Afrique de l'Ouest, Situation Actuelle, Expériences et Perspectives. FAO, GTZ, IDRC, CTA and STP/CIGQE: Rome.

Bambara D. 2011. Evaluation de la productivité céréalière des terres aménagées en demi-lunes, diguettes antiérosives et zaï dans le village de Tougou, nord du Burkina Faso. Mémoire de DEA, Univ. de Ouagadougou, Burkina Faso, 64 p.

Barro-Kondombo CP, Brocke KV, Chantereau J, Sagnard F, Zongo JD. 2008. Variabilité phénotypique des sorghos locaux de deux régions du Burkina Faso : la Boucle du Mouhoun et le Centre-Ouest. Cah. Agric., 17(2): 107-113.

Belem-Ouédraogo M. 1988. Recensement, Ecologie et Systématique des Asteraceae du Campus Universitaire d'AbidjanCocody. Univ. d'Abidjan : Côte d'Ivoire ; $96 \mathrm{p}$.

Busson F. 1965. Plantes alimentaires de l'Ouest Africain. Etude botanique, biologique et chimique. Ministère de la Coopération française : Marseille. 
CAPES (Centre d'Analyse des Politiques Economiques et Sociales). 2006. Etat des Lieux des Savoirs Locaux au Burkina Faso. $\quad$ CAPES/RGC-B PM: Ouagadougou ; 379 p.

CBD (Convention sur la Diversité Biologique). 2009. Quatrième rapport national sur la convention de la diversité biologique. ANDE, Côte d'Ivoire, 146 p. www.cbd.int/doc/world/ci/ci-nr-04-fr.doc (consulté le 24 novembre 2011)

Devineau J-L. 2001. Les espèces ligneuses indicatrices des sols dans les savanes et jachères de l'ouest du Burkina Faso. Phytocoenologia, 31(3): 325-351.

CTA (Centre Technique de Coopération Agricole et Rurale). 2009. Implications $d u$ Changement Climatique sur les Systèmes de Production Agricole Durables dans les Pays ACP; Quelles Stratégies? CTA: $108 \mathrm{p}$.

FAO (Food and Agriculture Organization). 2008. What is Agro-biodiversity? http://www.fao.org/docrep/007/y5609e/y5 609e01.htm (consulté le 22/11/2011).

FIDA (Fonds International de Développement Agricole). 2008. Le FIDA et le changement climatique. Consultation sur la huitième reconstitution des ressources du FIDA, REPL.VIII/4/R.10, Rome, 27 p.

Guinko S. 1984a. Action de l'homme dans la transformation de la végétation voltaïque par remaniement floristique. Bull. Trim. Inform. Sci., Note et Docum. Voltä̈ques, 15(4): 12-17.

Guinko S. 1984b. La végétation de la HauteVolta. Thèse de Doctorat ès Sciences Naturelles, Univ. de Bordeaux III, Bordeaux, 2 vol., 394 p.

Hahn-Hadjali K. 1997. Les groupements végétaux des savanes du sud-est du Burkina Faso (Afrique de l'Ouest). Etudes Flor. Veg., 3: 3-79.
INRAA-FAO. 2006. Deuxième rapport national sur l'état des resources phytogénétiques. INRAA, Algérie, 92 p. http://www.pgrfa.org/gpa/dza/algerie.pdf (consulté le 25 novembre 2011).

IPCC (Intergovernmental Panel on Climate Change). 2007. Climate Change: Impacts, Adaptation and Vulnerability. Working Group II: Contribution to the Fourth Assessment Report of the IPCC, Genève, $93 \mathrm{p}$.

INSD (Institut National de la Statique et de la Démographie). 2008. Recensement Général de la Population et de l'Habitation de 2006. MEF : Ouagadougou; $52 \mathrm{p}$.

Koussoubé A, Da DEC, Yacouba H, Karambiri H. 2006. Impact des techniques de conservation des eaux et des sols sur les rendements agricoles dans le bassin versant de Tougou. 2ie-AMMA, Ouagadougou, $5 \mathrm{p}$.

Lebrun JP, Toutain B, Gaston A, Boudet G. 1991. Catalogue des Plantes Vasculaires $d u$ Burkina Faso. IEMVT, CIRAD: France; $341 \mathrm{p}$.

Lo H, Kaere A. 2009. L'Afrique et les changements climatiques : enjeux et défis multiples. Agridape, 24(4): 6-7.

Mbayngone E, Thiombiano A, Hahn-Hadjali K, Guinko S. 2008. Caractéristiques écologiques de la végétation ligneuse du sud-est du Burkina Faso (Afrique de l'Ouest): cas de réserve de Pama. Candollea, 63: 17-33.

Naeem S, Defries R. 2009. La conservation des espèces, clé d'une adaptation climatique durable. In Adaptation au Changement Climatique, IEPF (ed). Liaison Énergie-Francophonie ; 117-121.

Nelson GC, Rosegrant MW, Koo J, Robertson R, Sulser T, Zhu T. 2009. Climate Change: Impact on Agriculture and Costs of Adaptation. IFPRI: Washington ; 19 p. 
Neumann K, Müller-Haude P. 1999. Forêts sèches au sud-ouest du Burkina Faso: végétation -sols - action de l'homme. Phytocoenologia, 29(1): 53-85.

Niang I. 2009. Le changement climatique et ses impacts: les prévisions au niveau mondial. In Adaptation au changement climatique, IEPF (ed). Liaison ÉnergieFrancophonie ; 13-20.

Ouédraogo A. 2006. Diversité et dynamique de la végétation ligneuse de la partie orientale du Burkina Faso. Thèse de doctorat, Univ. de Ouagadougou, 196 p.

Ouédraogo O. 2009. Phytosociologie, dynamique et productivité de la végétation du parc national d'Arly (Sudest du Burkina Faso). Thèse de Doctorat, Univ. de Ouagadougou, 188 p.

Paturel JE, Lubes H, Servat E, Delattre MO. 1996. Étude de séries pluviométriques de longue durée en Afrique de l'Ouest et Centrale non sahélienne. In Actes des XIIe Journées Hydrologiques de l'ORSTOM. ORSTOM : Montpellier; 8 p.
Sié M, Zongo JD, Dakouo D. 1998. Prospection des cultivars traditionnels de riz du Burkina Faso. Rev. CAMES, Sciences et Médecine, 21-27.

Soulama MA. 2010. La diversité biologique pour un développement durable. http://www.mediaterre.org/afrique-ouest/ actu,20081002185617.html (consulté le 20/07/2010).

Thiombiano A. 2005. Les combretaceae du Burkina Faso: taxonomie, écologie, dynamique et régénération des espèces. Thèse de Doctorat d'État ès Sciences Naturelles, Univ. de Ouagadougou, 290 p.

Traoré H. 1991. Influence des facteurs agroécologiques sur la constitution des communautés adventices des principales cultures céréalières (sorgho, mil, maïs) du Burkina Faso. Thèse de Doctorat ès Sciences Naturelles, Montpellier, France, $250 p$. 